\title{
Clinical relevance of non-tuberculous mycobacteria isolated in the Nijmegen-Arnhem region, The Netherlands
}

\author{
J van Ingen, ${ }^{1,2}$ S A Bendien, ${ }^{1}$ W C M de Lange, ${ }^{1}$ W Hoefsloot, ${ }^{1}$ P N R Dekhuijzen, ${ }^{1}$ \\ M J Boeree, ${ }^{1} D$ van Soolingen ${ }^{2}$
}

${ }^{1}$ Department of Pulmonary Diseases, Radboud University Nijmegen Medical Centre, Nijmegen, The Netherlands;

${ }^{2}$ National Mycobacteria Reference Laboratory, National Institute for Public Health and the Environment, Bilthoven, The Netherlands

Correspondence to: $\mathrm{Mr} \mathrm{J}$ van Ingen, National Institute for Public Health and the Environment (RIVM), National Mycobacteria Reference Laboratory (LIS: MYC pb22), PO Box 1, 3720 BA Bilthoven, The Netherlands: jakko.van.ingen@rivm.nl

Received 21 January 2009 Accepted 4 February 2009

Published Online First

12 February 2009

\begin{abstract}
Background: The frequency of clinical isolation of nontuberculous mycobacteria (NTM) in the Netherlands is increasing, but its clinical relevance is often uncertain.

Objective: To assess the frequency and clinical relevance of isolation of NTM in four associated hospitals in a single region in the Netherlands.
\end{abstract}

Methods: Medical files of all patients from whom NTM were isolated between January 1999 and January 2005 were reviewed retrospectively. Diagnostic criteria for nontuberculous mycobacterial disease published by the American Thoracic Society (ATS) were used to determine clinical relevance.

Results: 232 patients were found, from whom NTM were isolated from the respiratory tract in $91 \%$ of cases. Patients were mostly white men, with an average age of 60 years and pre-existing pulmonary disease. Fifty-three of 212 patients (25\%) with pulmonary isolates met the ATS diagnostic criteria for pulmonary NTM disease; this percentage differed by species. Most patients were treated with rifampicin, ethambutol and clarithromycin. Treatment outcome for pulmonary NTM disease was suboptimal but differed by species: overall, improvement was seen in $67 \%$ of treated patients, but in only $50 \%$ of those with pulmonary $M$ avium disease. Lymphadenitis was the most common extrapulmonary disease type.

Conclusions: Twenty-five per cent of all patients with pulmonary NTM isolates met the ATS criteria. Clinical relevance differs by species. NTM isolation increases over time. Species distribution differs from that of neighbouring countries and the $M$ avium complex isolates have traits different from those reported in the USA. Adherence to diagnostic and treatment guidelines can be improved.

The clinical isolation of non-tuberculous mycobacteria (NTM) increases in many countries where the incidence of tuberculosis is decreasing, with marked geographical differences in the species encountered. ${ }^{1-3}$ The NTM are often opportunistic pathogens, capable of causing disease in patients with impaired immunity, either local due to preexisting pulmonary disease or systemic-for example, haematological malignancy, immunosuppressive drug treatment or HIV/AIDS. ${ }^{1}$ The clinical relevance of isolated NTM is often unclear. The NTM are common in the environment and can survive in flowing water systems. ${ }^{4}$ Moreover, NTM resist common disinfectants. ${ }^{5}$ Thus, pseudo-infection due to occasional presence of NTM in clinical samples as a consequence of contamination of medical tools should always be considered. ${ }^{167}$
To differentiate true infection from pseudoinfection, and establish the clinical relevance of an NTM isolate, is of paramount importance since treatment of NTM disease is time consuming and often complicated. To assist in this differentiation, the American Thoracic Society (ATS) established general criteria for the diagnosis of pulmonary nontuberculous mycobacterial infection (box 1). ${ }^{1}$

We performed a retrospective case study to assess the prevalence and clinical relevance of NTM isolated in the four associated training hospitals of the Nijmegen-Arnhem region, the Netherlands, using the ATS diagnostic criteria to differentiate NTM disease from pseudo-infection or contamination.

\section{METHODS}

To determine clinical relevance, we examined medical records of all inpatients and outpatients in four collaborating hospitals in the NijmegenArnhem region of the Netherlands from whom NTM were isolated between January 1999 and January 2005. We recorded demographic, clinical and microbiological data and status according to the diagnostic criteria for pulmonary NTM disease by the ATS. ${ }^{1}$ We considered pulmonary isolates clinically relevant if the patient met the ATS diagnostic criteria in the absence of evidence of other pulmonary infections.

For patients treated for NTM disease, we defined cure as symptomatic improvement and reversion to negative cultures sustained throughout our follow-up period. We focused on pulmonary isolates.

The Dutch National Institute for Public Health and the Environment (RIVM) subjected isolates of all patients to laboratory diagnosis. The RIVM is the national reference laboratory that provides identification, drug susceptibility testing and genotyping of mycobacterial isolates for all hospitals in the Netherlands. To identify NTM, the INNOLiPA MYCOBACTERIA v2 (Innogenetics, Gent, Belgium) reverse line blot assay was used, after ruling out membership of the $M$ tuberculosis complex using a Hain GenoType MTBC line probe assay (Hain Lifescience, Nehren, Germany). If no species-specific result was obtained, 16S rDNA gene sequencing (151 bp hypervariable region A) was performed. Before 2004, 16S rDNA gene sequencing was performed, after ruling out membership of the $M$ tuberculosis complex or $M$ avium complex using the AccuProbe MTB and $M$ avium complex DNA probe kits (GenProbe, San Diego, 
Box 1: Summary of the American Thoracic Society diagnostic criteria for pulmonary non-tuberculous mycobacterial infection'

\section{Clinical}

- Pulmonary symptoms, nodular or cavitary opacities on chest radiograph, or a high-resolution CT scan that shows multifocal bronchiectasis with multiple small nodules.

and

- Appropriate exclusion of other diagnoses.

Microbiological

- Positive culture results from at least two separate expectorated sputum samples. (If the results from the initial sputum samples are non-diagnostic, consider repeat sputum acid-fast bacillus (AFB) smears and cultures.)

or

- Positive culture results from at least one bronchial wash or lavage.

or

- Transbronchial or other lung biopsy with mycobacterial histopathological features (granulomatous inflammation or AFB) and positive culture for non-tuberculous mycobacteria (NTM) or biopsy showing mycobacterial histopathological features (granulomatous inflammation or AFB) and one or more sputum or bronchial washings that are culture positive for NTM.

- Expert consultation should be obtained when NTM are recovered that are either infrequently encountered or that usually represent environmental contamination.

- Patients who are suspected of having NTM lung disease but who do not meet the diagnostic criteria should be followed up until the diagnosis is firmly established or excluded.

- Making a diagnosis of NTM lung disease does not, of itself, necessitate the institution of treatment, which is a decision based on potential risks and benefits of treatment for individual patients.

USA). Sequencing results were compared with the GenBank (National Center for Biotechnology Information, http://www. ncbi.nlm.nih.gov (accessed 27 March 2009) database. A Pearson $\chi^{2}$ test was used for statistical correlations. The regional ethics committee approved the study.

\section{RESULTS}

We found 232 patients with NTM isolates, mostly from the respiratory tract $(n=212,91 \%)$. Primary isolates were cultured from sputum $(\mathrm{n}=158 ; 75 \%)$, bronchoalveolar lavage samples $(\mathrm{n}=47 ; 22 \%)$ and lung biopsy specimens $(\mathrm{n}=7 ; 3 \%)$. The annual number of patients from whom NTM were isolated rose during our research period, mainly owing to an increase in pulmonary isolates, especially $M$ avium complex (MAC) isolates (fig 1). The annual percentage of patients meeting the ATS criteria did not change.

Table 1 presents the baseline characteristics of the 212 patients with pulmonary NTM isolates.

Fifty-three patients (25\%) met the ATS diagnostic criteria and were thus likely to have pulmonary NTM disease. Of the 159 patients who did not meet the ATS criteria, $146(92 \%)$ failed to meet microbiological criteria and 149 (94\%) failed to meet radiological criteria. For these patients, follow-up for a mean

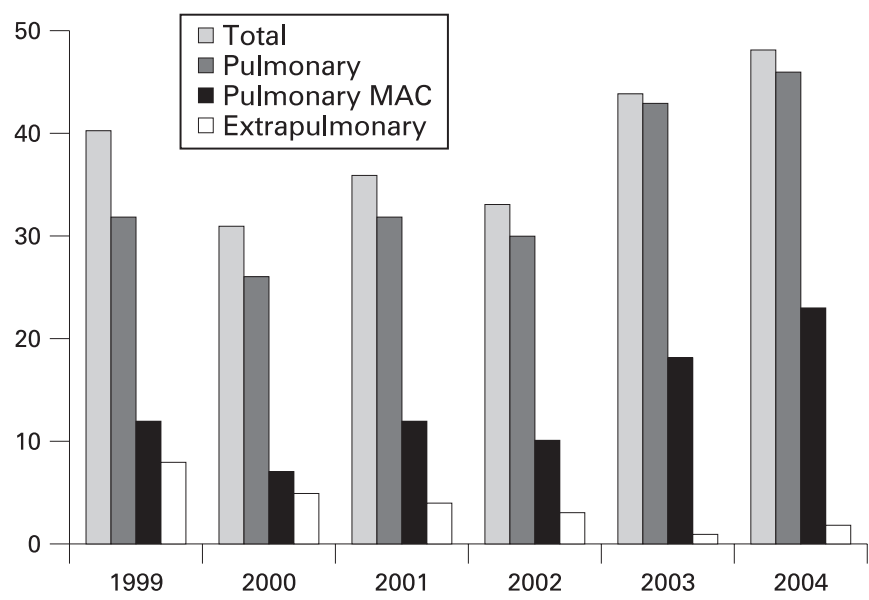

Figure 1 Total number of patients with non-tuberculous mycobacteria isolates, per year and isolation site. MAC, $M$ avium complex.

duration of 47 months (range 29-98 months) did not yield additional evidence of NTM disease.

Figure 2 visualises the clinical relevance of the various NTM species, defined by the percentage of patients who met the ATS diagnostic criteria. Four cases of pulmonary NTM disease were actually relapses of disease episodes before our study period (two $M$ avium, one $M$ xenopi, one $M$ kansasii).

Eighty-five patients (40\%) had MAC isolates (59 M avium, 16 $M$ intracellulare, 10 other MAC) of whom 26 (24 $M$ avium, $2 M$ intracellulare; $31 \%$ ) met the ATS diagnostic criteria. $M$ avium isolation was clinically more relevant than $M$ intracellulare $(24 / 59=41 \%$ vs $2 / 16=13 \%$ of patients met the ATS diagnostic criteria). A radiographic presentation with fibrosis and cavities was more prevalent than nodular bronchiectatic disease (12 vs 3 cases for $M$ avium and one case each for $M$ intracellulare).

Whereas MAC was most frequently seen, the degree of clinical relevance was higher for isolation of $M$ malmoense (2/3), $M$ kansasii (12/17, 71\%), $M$ xenopi (3/5), $M$ szulgai (4/4), $M$ celatum (2/2) and $M$ genavense (1/1; fig 2).

Symptoms recorded at presentation varied widely; only fever $(p=0.014, O R=2.422,95 \%$ CI 1.179 to 4.978$)$ and fatigue $(p<0.001, O R=3.477,95 \%$ CI 1.721 to 7.024$)$ at presentation were associated with meeting the ATS diagnostic criteria (table 1). Chest radiographs were performed for 206 of the 212 patients and abnormalities were noted in 161 patients (78\%; table 1). Abnormalities did not differ significantly by NTM species. Three patients with pulmonary NTM disease presented with a pulmonary mass, mimicking malignancy. Computed tomography (CT) scans were performed in 107 patients (50\%); among patients meeting the ATS diagnostic criteria, five cases of fibrocavitary disease and six cases of nodular-bronchiectatic disease were noted in patients with inconclusive chest radiographs. On chest radiographs, these cases of fibrocavitary disease presented with dense air-space opacities; the nodularbronchiectatic disease appeared as coarse linear scarring.

Drug treatment for mycobacterial infection was started in 44 patients; 31 started treatment for tuberculosis (TB) and switched to an NTM treatment regimen, 13 patients received standard TB treatment only. Of these 44 patients, 30 met the 1997 ATS diagnostic criteria available during the study period. ${ }^{8}$ Among those 30, 20 (67\%) were cured, defined by symptomatic improvement and reversion to negative cultures, five died during treatment (three with $M$ avium, one with $M$ intracellulare, one with $M$ xenopi disease; 17\%) and five had a relapse after 
Table 1 Baseline characteristics of patients with pulmonary nontuberculous mycobacteria (NTM) isolates

\begin{tabular}{lllc}
\hline Characteristics & $\begin{array}{l}\text { ATS }+ \\
(\mathbf{n}=\mathbf{5 3})\end{array}$ & $\begin{array}{l}\text { ATS }- \\
(\mathbf{n}=\mathbf{1 5 9})\end{array}$ & $\begin{array}{l}\text { Total } \\
(\mathbf{n}=\mathbf{2 1 2})\end{array}$ \\
\hline Demography & & & \\
$\quad$ Male & $37(70)$ & $95(60)$ & $132(62)$ \\
$\quad$ Mean age (years) & 57 & 61 & 60 \\
$\quad$ Dutch origin & $48(91)$ & $145(91)$ & $193(91)$
\end{tabular}

Predisposing conditions

$\begin{array}{lccc}\text { Pre-existing pulmonary disease } & 37(70) & 125(79) & 162(76) \\ \text { COPD } & 23(43) & 80(50) & 103(49) \\ \text { Lung cancer } & 4(8) & 17(11) & 21(10) \\ \text { Healed tuberculosis } & 6(11) & 22(14) & 28(13) \\ \text { Previous pulmonary NTM disease } & 4(8) & 0 & 4(2) \\ \text { Asthma } & 4(8) & 21(13) & 25(12) \\ \text { Bronchiectasis } & 8(15) & 8(5) & 16(8) \\ \text { Current or past smoker } & 35(66) & 94(59) & 129(61) \\ \text { Alcohol abuse } & 8(15) & 16(10) & 24(11) \\ \text { High-dose steroid use } & 2(4) & 6(4) & 8(4) \\ \text { HIV infection } & 6(11) & 7(4) & 13(6) \\ \text { Haematological malignancy } & 1(2) & 4(3) & 5(2) \\ \text { Anti-TNF treatment } & 1(2) & 1(1) & 2(1) \\ \text { Otherwise impaired immunity } & 3 \dagger & 3 \ddagger & 6\end{array}$

Symptoms

$\begin{array}{lcrr}\text { Productive cough } & 40(75) & 126(79) & 166(78) \\ \text { Haemoptysis } & 10(19) & 28(18) & 38(18) \\ \text { Dyspnoea } & 29(55) & 85(54) & 114(54) \\ \text { Fever } & 19(36) \S & 30(19) & 49(23) \\ \text { Night sweats } & 7(13) & 16(10) & 23(11) \\ \text { Weight loss } & 14(26) & 29(18) & 43(20) \\ \text { Fatigue } & 28(53) \uparrow & 37(23) & 65(31)\end{array}$

Chest radiograph features

Air-space opacities

Cavity

Nodules

Bronchiectasis

Emphysema

$22(42)$

$15(28)$

4 (8)

$4(8)$

$14(26)$

$42(26)$

2 (1)

2 (1)

$5(3)$

$23(15)$

$64(30)$

17 (8)

6 (3)

$9(4)$

37 (17)

Microbiology

Mean number of cultures performed

Mean number of positive cultures

AFB smear positive

$\begin{array}{clc}4.9 & 3.5 & 3.8 \\ 2.9 & 1.1 & 1.5 \\ 26(49)^{* *} & 6(4) & 32(15)\end{array}$

Results are shown as $\mathrm{n}(\%)$ unless stated otherwise.

*>15 mg prednisone/day for $>3$ months before primary NTM culture; †anorexia nervosa, lymphopenia and complement C4 deficiency, diabetes mellitus (all $n=1)$; tintravenous drug abuse $(n=2)$, azathioprine use $(n=1) ; \xi p=0.014,0 R=2.422$, $95 \% \mathrm{Cl} 1.179$ to $4.978 ;-9 \mathrm{p}<0.001, \mathrm{R}=3.477,95 \% \mathrm{Cl} 1.721$ to $7.024 ;{ }^{* *} \mathrm{p}<0.001$, $\mathrm{OR}=23.583,95 \% \mathrm{Cl} 8.777$ to 63.125 .

AFB, acid-fast bacillus; COPD, chronic obstructive pulmonary disease; TNF, tumour necrosis factor.

treatment (four with $M$ avium, one with $M$ celatum disease; 17\%) The mean interval between the end of treatment and culture-proven relapse was 31 months (range 23-44). Of 14 patients treated for pulmonary $M$ avium disease, only seven improved. The regimens used mostly consisted of rifampicin and ethambutol, despite frequent in vitro resistance $(15 / 25$ primary isolates were resistant to rifampicin, $18 / 25$ to ethambutol; five were not tested); isoniazid was added in 11 patients (despite in vitro resistance in all cases), clarithromycin in 19, ciprofloxacin in four. Clarithromycin and ciprofloxacin were used only in patients with susceptible isolates. Three patients received 6 months of standardised TB treatment only. The mean treatment duration was 13 months (range 1-37). Three patients received additional surgical treatment that consisted of resection of a solitary pulmonary mass in two and lobectomy for persisting fibrocavitary disease in one; all three were cured. The mean duration of follow-up after treatment was 34 months (range 17-91).

Treatment was not given in 168 patients, though eight of them met the 1997 ATS diagnostic criteria. ${ }^{8}$ Four patients were considered to have too few symptoms or radiographic deterioration, one refused treatment and one patient spontaneously reversed to negative cultures. Slow radiographic deterioration of pulmonary disease was apparent in all seven persistently symptomatic and culture-positive, untreated patients.

During the study period, NTM were isolated from extrapulmonary samples only of 20 patients. Lymphadenitis was the most common extrapulmonary NTM disease type; six children and one elderly woman had cervical lymphadenitis (three $M$ avium, two $M$ malmoense, one $M$ intracellulare, one $M$ haemophilum) and one patient with AIDS (CD4 count 1) had axillar and mediastinal lymphadenitis with extensive abscess formation, caused by $M$ gordonae. We recorded three cases of skin infection: two caused by $M$ marinum in fish tank owners and one by $M$ malmoense in a patient with haematological malignancy. We also noted single cases of $M$ kansasii tenosynovitis in a gardener, $M$ abscessus otomastoiditis after tympanostomy tube placement in a child and disseminated $M$ avium disease in an AIDS patient (CD4 count 40). The remaining six patients had MAC isolated from the digestive tract, in the absence of further evidence of NTM disease.

\section{DISCUSSION}

Twenty-five per cent of all patients in this study had pulmonary NTM disease according to the ATS diagnostic criteria, and the clinical relevance differed significantly by species (fig 2). Isolation of $M$ malmoense, $M$ xenopi, $M$ szulgai, $M$ kansasii, $M$ celatum and $M$ genavense warrants special attention, as this usually reflects true NTM disease; an observation confirmed in previous studies. ${ }^{1}$ Although in the current study the number of isolates of many species is too low to permit firm conclusions, similar rates of clinical relevance have already emerged for $M$ xenopi (21/45 patients; 47\%), $M$ chelonae (7/35; 20\%), $M$ abscessus (13/39; 33\%), $M$ simiae (3/28; 11\%) and $M$ szulgai $(11 / 15 ; 73 \%)$ in nationwide studies of pulmonary isolates in the Netherlands. ${ }^{9-12}$ These data and the additional data in our current study strengthen a presentation such as that shown in fig 2 . The $25 \%$ of patients who met the ATS criteria is similar to the $33 \%$ recently found in a Canadian referral centre study, 25\% recorded among patients in Korea and the results of earlier studies reviewed by Marras and Daley. ${ }^{2} 1314$

A difference in clinical relevance by species has also been noted in the recently published Korean study with results similar to ours, except for a very limited relevance of $M$ szulgai (2/32 patients; 6\%) and $M$ celatum (1/11; 9\%). ${ }^{13}$ These differences in clinical relevance between NTM species emphasise the major role of the bacteriological laboratory in the management of these emerging infections; correct identification of NTM isolates is a prerequisite for correct patient handling. These apparent regional differences in clinical relevance of NTM species require further study.

The ATS diagnostic criteria are based on experience with $M$ avium, $M$ kansasii and $M$ abscessus ${ }^{1}$; their applicability to less studied species may be limited. ${ }^{19}$ The clinical applicability of the ATS criteria is supported by those patients who did not meet the ATS criteria and who had no clinical event or new radiological events during follow-up. Very few follow-up 
Figure 2 Clinical relevance of pulmonary non-tuberculous mycobacteria isolates in this study, per species. $(x / y)$, number of patients who met the American Thoracic Society diagnostic criteria/total number of patients per species.

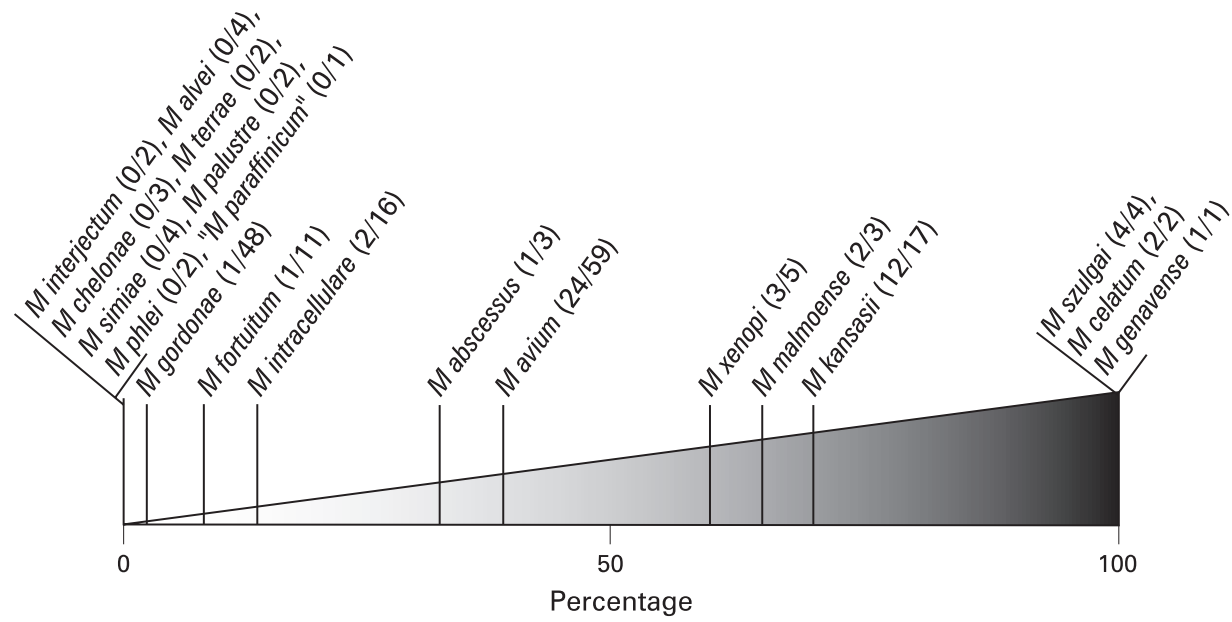

cultures were performed (table 1), although this was advocated in the ATS statement available during the study period. ${ }^{8}$

Although for some species the small number of isolates decreases the weight of the conclusions of the analysis, the difference in clinical relevance of the various NTM species may mean that for the species to the left of fig 2 the ATS criteria should be very strictly applied or even be made more stringent, whereas among the upper half (50-"100"\% clinical relevance) a diagnosis of NTM disease may be justified after the first positive culture.

The increase in the annual number of patients from whom NTM were isolated (fig 1) is a cause for concern and has also been noted in previous studies. ${ }^{12} 1315$ This increase was often thought be related to improvements in laboratory techniques; in the Netherlands, the use of (automated) liquid culture systems increased during the study period. ${ }^{1}{ }^{16}$ A recent study, however, demonstrated that skin sensitisation to $M$ intracellulare has also increased significantly over recent decades in the USA, consistent with observed increases in the rate of pulmonary NTM infections. ${ }^{17}$ Several aspects may influence this increase in NTM isolation. First, the Dutch population, as in many developed countries, is ageing and an increasing prevalence of chronic obstructive pulmonary disease is observed. ${ }^{18-20}$ The baseline characteristics of our study group, predominantly men with an average age of 60 years, similar to previous NTM studies in societies with low HIV prevalence, ${ }^{113}{ }^{15}$ reflect these changes. Second, for chronic inflammatory diseases, immunosuppressive drugs, including tumour necrosis factor $\alpha$ neutralising agents, are increasingly used. Both these aspects may have an impact on the future prevalence of NTM disease. In addition, the exposure to aerosols of tap water may have increased over time, as frequency of showering has risen dramatically over recent decades. The minor role of HIV infection probably reflects the low prevalence of HIV infection in the Netherlands, compared with chronic pulmonary disease, and the advent of highly active antiretroviral treatment. This limits the number of cases of severe HIV immunosuppression and its co-infections, ${ }^{21}$ which may be reflected in the decreasing NTM isolation from extrapulmonary samples (fig 1). Besides, the hospitals participating in the study are situated in the eastern part of the Netherlands, where HIV incidence is lower than in the more urbanised western part..$^{22}$

The limited associations between symptoms, chest radiography results and NTM disease according to the ATS diagnostic criteria underline the fact that diagnosis of NTM remains difficult. Hence, the NTM merit special attention by doctors and microbiologists.

Despite inherent limitations, the species found in this study and their frequencies probably reflect the current epidemiological situation for NTM in the Netherlands. This situation is remarkably different from nearby southeast England, where $M$ xenopi is most common and Scotland and Scandinavia, where $M$ malmoense is especially prevalent. ${ }^{1423}$ The species distribution and clinical relevance among MAC isolates seen in this study differs from recent reports from the USA where $M$ intracellulare is the more common respiratory pathogen, ${ }^{1}$ clinically more relevant than $M$ avium in non-HIV patients, ${ }^{24}$ and nodularbronchiectatic pulmonary MAC disease, associated with postmenopausal immunocompetent women, is as prevalent as cavitary disease..$^{24}$ Possibly, the predominance of $M$ avium and cavitary disease in our study group are inter-related; different MAC subtypes are known to cause different disease types in humans. ${ }^{25}$ Cases of nodular-bronchiectatic disease may have also been missed owing to the infrequent use of CT scanning and small number of follow-up cultures performed; diagnosing this paucibacillary disease type generally demands prolonged, intense follow-up. ${ }^{1}$ Alternatively, since we used hospital inpatient and outpatient file review, cases of nodular-bronchiectatic disease may have been missed as they are less often referred to hospitals for diagnosis, owing to a more indolent clinical course ${ }^{1}$ compared with cavitary MAC disease.

Previous studies have recorded high degrees of clinical relevance of $M$ kansasii, comparable to the $71 \%$ in this study. ${ }^{14}$ Whereas the latest ATS statement states that for $M$ kansasii the treatment decision may be based on a single positive culture in select cases, ${ }^{1}$ we recorded single positive sputum cultures in five patients with non-suspect radiographic changes and repeatedly negative followup cultures. This would have meant an unnecessary treatment for these patients when applying the new ATS criteria.

We observed possible instances of both undertreatment (not treating those who meet the diagnostic criteria) and overtreatment (treatment of those who do not meet diagnostic criteria), as well as the use of treatment regimens that have never proved to be effective. This may harm patients. This, as well as the fact that few (high-resolution) CT scans and followup cultures, both strongly advocated in the ATS criteria, ${ }^{18}$ were performed in patients with NTM isolates in non-cavitary pulmonary disease, could reflect a lack of knowledge of and experience with the diagnosis and management of NTM disease 
in doctors, or clinical circumstances not captured in our file review. All these aspects emphasise the need for centralisation of knowledge, experience and care for patients with (suspected) NTM disease or increased expert consultation.

Treatment duration recorded in our study was not in accordance with the British Thoracic Society (BTS) and ATS guidelines. ${ }^{126}$ A favourable outcome for $67 \%$ of patients with pulmonary NTM disease is higher than the $36 \%$ observed in the recently published BTS clinical trial comparing regimens with clarithromycin or ciprofloxacin as adjuncts to rifampicin and ethambutol. ${ }^{27}$ In that trial, patients with MAC and $M$ xenopi had worse outcomes than those with $M$ malmoense. ${ }^{27}$ The differences in treatment outcome between our study and the BTS trial may result, first, from our more lenient definition of "cure", second, our limited follow-up period and finally, the NTM species causing pulmonary disease in our patients. Whereas the first trials of macrolide-based regimens in pulmonary MAC disease in HIV-negative patients in the USA reported cure rates of 59-92\%, with comparable definitions of cure, ${ }^{1}$ the outcome of treatment for pulmonary MAC disease seen in our study and the BTS trial was disappointingly poorer. The treatment results, over-representation of men and cavitary disease, MAC species distribution and clinical relevance all suggest differences in MAC organisms encountered in the USA and Europe.

Although, generally, outcome of NTM treatment is suboptimal, ${ }^{1}$ various factors may have influenced our results. First, the conditions predisposing to NTM disease influence the outcome of the patient. Second, the 1997 ATS diagnostic criteria $^{8}$ available to doctors in the study period might not have selected the group of patients who would have benefited from treatment. More patients meet the recently published criteria, ${ }^{12}$ though herein it is stated that meeting the criteria does not of itself necessitate treatment. ${ }^{1}$ Still, this may lead to improved selection of patients to receive treatment and thus improve outcome rates. In this perspective, the term under-treatment will become invalid. Third, previous trials for pulmonary MAC disease have recorded better treatment outcome using similar definitions, but in a different MAC population. In our group more patients had cavitary disease, which in general is associated with a worse treatment outcome. ${ }^{1}$ Finally, one of the centres incorporated in this study is a referral centre for NTM disease treatment, which may create a selection bias.

Extrapulmonary disease was rare, though its overall frequency $(9 \%)$ and distribution of disease types are in line with previous studies, summarised in the recent ATS statement. ${ }^{1}$

In conclusion, clinical isolation of NTM was relevant in $25 \%$ of the 212 patients with pulmonary isolates. Clinical relevance differs markedly by species and there are important regional differences in species distribution and relevance. This, as well as the rising isolation rates of NTM, warrants special attention by doctors and microbiologists. Treatment outcome of pulmonary NTM disease was disappointing. Both overtreatment and insufficient treatment were noted. Extrapulmonary NTM disease is rare. Actions to increase awareness of the ATS diagnostic criteria and management guidelines and centralisation of NTM disease management are strongly recommended.

\section{Competing interests: None.}

Ethics approval: Regional ethics committee approval obtained.

\section{REFERENCES}

1. Griffith DE, Aksamit T, Brown-Elliot BA, et al. An official ATS/IDSA statement: diagnosis, treatment, and prevention of nontuberculous mycobacterial diseases. Am J Respir Crit Care Med 2007;175:367-416.

2. Marras TK, Chedore P, Ying AM, et al. Isolation prevalence of pulmonary nontuberculous mycobacteria in Ontario, 1997-2003. Thorax 2007;62:661-6.

3. Martín-Casabona N, Bahrmand AR, Bennedsen J, et al. Non-tuberculous mycobacteria: patterns of isolation. A multi-country retrospective survey. Int J Tuberc Lung Dis 2004;8:1186-93.

4. Torvinen E, Lehtola MJ, Martikainen PJ, et al. Survival of Mycobacterium avium in drinking water biofilms as affected by water flow velocity, availability of phosphorus, and temperature. Appl Environ Microbiol 2007;73:6201-7.

5. Dauendorffer JN, Laurain C, Weber M, et al. Evaluation of the bactericidal efficiency of a $2 \%$ alkaline gluteraldehyde solution on Mycobacterium xenopi. J Hosp Inf 2000;46:73-6.

6. Astagneau P, Desplaces N, Vincent V, et al. Mycobacterium xenopi spinal infections after discovertebral surgery: investigation and screening of a large outbreak. Lancet 2001;358:747-51.

7. Portaels F. Epidemiology of mycobacterial diseases. Clin Dermato/ 1995;13:207-22.

8. Wallace RJ Jr, Glassroth J, Griffith DE, et al. American Thoracic Society. Diagnosis and treatment of disease caused by nontuberculous mycobacteria. Am J Respir Crit Care Med 1997;156:S1-25.

9. van Ingen J, Boeree MJ, Dekhuijzen PNR, et al. Clinical relevance of Mycobacterium simiae in pulmonary samples. Eur Respir J 2008;31:106-9.

10. van Ingen J, Boeree MJ, de Lange WCM, et al. Clinical relevance of Mycobacterium szulgai in the Netherlands. Clin Infect Dis 2008;46:1200-5.

11. van Ingen J, Boeree MJ, de Lange WCM, et al. Clinical relevance of Mycobacterium xenopi isolation and its determinants in the Netherlands. Emerg Infect Dis 2008;14:385-9

12. van Ingen J, Boeree MJ, de Lange WC, et al. Impact of new American Thoracic Society diagnostic criteria on management of nontuberculous mycobacterial infection. Am J Respir Crit Care Med 2007;176:418.

13. Koh WJ, Kwon OJ, Jeon K, et al. Clinical significance of nontuberculous mycobacteria isolated from respiratory specimens in Korea. Chest 2006;129:341-8.

14. Marras TK, Daley CL. Epidemiology of human pulmonary infection with nontuberculous mycobacteria. Clin Chest Med 2002;23:553-67.

15. Henry MT, Inamdar L, O'Riordain D, et al. Nontuberculous mycobacteria in non-HIV patients: epidemiology, treatment and response. Eur Respir J 2004;23:741-6.

16. Donnabella V, Salazar-Schicchi J, Bonk S, et al. Increasing incidence of Mycobacterium xenopi at Bellevue Hospital. An emerging pathogen or a product of improved laboratory methods? Chest 2000;118:1365-70.

17. Khan K, Wang J, Marras TK. Nontuberculous mycobacterial sensitization in the United States: national trends over three decades. Am J Respir Crit Care Med 2007;176:306-13.

18. Murray CJL, Lopez AD. The global burden of disease: a comprehensive assessment of mortality and disability from diseases, injuries and risk factors in 1990 and projected to 2020. Cambridge: Harvard School of Public Health, 1996.

19. Tirimanna PR, van Schayck CP, den Otter JJ, et al. Prevalence of asthma and COPD in general practice in 1992: has it changed since 1977? Br Gen Pract 1996:46:277-81.

20. World Health Organization. Health topics: Ageing and life course. Available at http://www.who.int/ageing/en (accessed 26 March 2009).

21. Karakousis PC, Moore RD, Chaisson RE. Mycobacterium avium complex in patients with HIV infection in the era of highly active antiretroviral therapy. Lancet Infect Dis 2004; 4:557-65.

22. Dukers NH, Fennema HS, van der Snoek EM, et al. HIV incidence and HIV testing behavior in men who have sex with men: using three incidence sources, The Netherlands, 1984-2005. AIDS 2007;21:491-9.

23. Yates MD, Pozniak A, Uttley AHC, et al. Isolation of environmental mycobacteria from clinical specimens in South-East England 1973-1993. Int J Tuberc Lung Dis 1997;:1:75-80.

24. Han $\mathbf{X Y}$, Tarrand JJ, Infante R, et al. Clinical significance and epidemiologic analyses of Mycobacterium avium and Mycobacterium intracellulare among patients without AIDS. J Clin Microbiol 2005;43:4407-12

25. Smole SC, McAleese F, Ngampasutadol J, et al. Clinical and epidemiological correlates of genotypes within the Mycobacterium avium complex defined by restriction and sequence analysis of hsp65. J Clin Microbiol 2002;40:3374-80.

26. Subcommittee of the Joint Tuberculosis Committee of the British Thoracic Society. Management of opportunist mycobacterial infections: Joint Tuberculosis Committee guidelines 1999. Thorax 2000;55:210-8

27. Research Committee of the British Thoracic Society. Clarithromycin vs ciprofloxacin as adjuncts to rifampicin and ethambutol in the treatment of opportunist mycobacterial pulmonary diseases and an assessment of the value of immunotherapy with $M$ vaccae: a pragmatic, randomised trial by The British Thoracic Society. Thorax 2008:63:627-34. 